J. Hejduk, Institute of Mathematics, Łodz University, ul. Banacha 22, $90-238$ Łodz, Poland

A. Kharazishvili, Institute of Applied Mathematics, University of Tbilisi, University str. 2, 380043 Tbilisi 43, Georgia

\title{
ON DENSITY POINTS WITH RESPECT TO VON NEUMANN'S TOPOLOGY
}

\begin{abstract}
The paper is devoted to a topology of von Neumann type which is associated with a special invariant extension of the classical Lebesgue measure. The structure of the sets of density points for some sets having the Baire property with respect to this topology is investigated. In particular, a problem of Wilczynski concerning density points in the sense of category is solved.
\end{abstract}

\section{Introduction}

Let $\mathbb{R}$ be the real line and let $l$ be the classical Lebesgue measure on $\mathbb{R}$. If we want to estimate how big a Lebesgue measurable set $X \subseteq \mathbb{R}$ is near a point $x \in \mathbb{R}$, then we consider the real number

$$
d(X, x)=\lim _{h \rightarrow 0^{+}}(l(X \cap[x-h, x+h]) / 2 h)
$$

and call it the density of the set $X$ at the point $x$. If $d(X, x)=1$, then we simply say that $x$ is a Lebesgue density point of $X$. Hence we may put

$$
\Phi_{d}(X)=\{x \in \mathbb{R}: d(X, x)=1\}
$$

for each Lebesgue measurable subset $X$ of $\mathbb{R}$. We can also consider the class of sets

$$
T_{d}=\left\{X \subseteq \mathbb{R}: X \subseteq \Phi_{d}(X)\right\} .
$$

Key Words: von Neumann's topology, Wilczynski's topology, convergence in measure, convergence almost everywhere, density point, the Lebesgue measure, invariant extension of measure, extension of topology, almost invariant partition, thick set

Mathematical Reviews subject classification: Primary: 28A05, 28D05

Received by the editors June 3, 1995 
It is well known that this class of sets is a topology on the set $\mathbb{R}$ extending the usual Euclidean topology of $\mathbb{R}$. The topology $T_{d}$ is called the density topology on $\mathbb{R}$. (See, for example, [1] where some interesting properties of this topology are considered; see also [2] where some additional properties of the density topology are discussed, as well.)

It is not difficult to check that 0 is a density point of a Lebesgue measurable set $X \subseteq \mathbb{R}$ if and only if

$$
\lim _{n \rightarrow \infty} n l(X \cap[-1 / n, 1 / n])=2 .
$$

Obviously, this is equivalent to saying that $\lim _{n \rightarrow \infty} l((n X) \cap[-1,1])=2$ where $n X$ denotes the set $\{n x: x \in X\}$. The last relation means that the sequence of characteristic functions

$$
\left\{\chi_{n X} \cap[-1,1]: n \in \mathbb{N}\right\}
$$

tends in measure to the characteristic function of the segment $[-1,1]$. By the well-known Riesz theorem from measure theory, we can describe the convergence in measure in terms of the convergence almost everywhere. This simple observation inspired W. Wilczynski to introduce in 1982 the concept of a density point with respect to category (see [3] or [5]).

Let $T$ be a topology on $\mathbb{R}$ satisfying the following three conditions:

(1) the segment $[-1,1]$ has the Baire property with respect to $T$,

(2) for every positive integer $n$ and for each set $X$ having the Baire property with respect to $T$, the set $n X$ also has the Baire property with respect to $T$,

(3) for each translation $h$ of $\mathbb{R}$ and for each set $X$ having the Baire property with respect to $T$, the set $h+X$ also has the Baire property with respect to $T$.

Notice that all the topologies $T$ considered below are assumed, as a rule, to satisfy these three conditions. Notice also that conditions (1) and (3) imply that all Borel subsets of $\mathbb{R}$ have the Baire property with respect to $T$. Let $B(\mathbb{R}, T)$ denote the $\sigma$-algebra of all sets with the Baire property with respect to the topology $T$ and let $K(\mathbb{R}, T)$ denote the $\sigma$-ideal of all first category sets with respect to $T$. Let $X$ be an arbitrary set from the $\sigma$-algebra $B(\mathbb{R}, T)$. We shall say that 0 is a $K(\mathbb{R}, T)$-density point of the set $X$ if the sequence of characteristic functions $\left\{f_{n}: n \in \mathbb{N}\right\}=\left\{\chi_{n X} \cap[-1,1]: n \in \mathbb{N}\right\}$ converges to $\chi_{[-1,1]}$ with respect to the ideal $K(\mathbb{R}, T)$. The last sentence means that 0 is a $K(\mathbb{R}, T)$-density point of $X$ if and only if, for each infinite subset $N_{1}$ of $\mathbb{N}$, 
there exists an infinite subset $N_{2}$ of $N_{1}$ such that the corresponding partial sequence of functions $\left\{f_{n}: n \in N_{2}\right\}$ converges to $\chi_{[-1,1]}$ on the complement of a member of $K(\mathbb{R}, T)$ (in other words, this partial sequence of functions converges $K(\mathbb{R}, T)$-almost everywhere).

Now, let $x$ be an arbitrary point of $\mathbb{R}$. We shall say that $x$ is a $K(\mathbb{R}, T)$ density point of the set $X$ if 0 is a $K(\mathbb{R}, T)$-density point of the translated set $\{y-x: y \in X\}$.

For any set $X$ having the Baire property with respect to the topology $T$, let us put $\Phi_{T}(X)=$ the set of all $K(\mathbb{R}, T)$-density points of $X$. In this way we obtain the following family of sets:

$$
T^{*}=\left\{X \in B(\mathbb{R}, T): X \subseteq \Phi_{T}(X)\right\} .
$$

If the family $T^{*}$ forms a topology on the set $\mathbb{R}$, then this topology is called the Wilczynski topology on $\mathbb{R}$ associated with $T$ or the $K(\mathbb{R}, T)$-density topology on $\mathbb{R}$ (see [3]).

Example 1 Let us consider a particular case of the above construction. Namely, let us take as $T$ the usual Euclidean topology on $\mathbb{R}$. In this case, $T^{*}$ is the topology on $\mathbb{R}$ called the I-density topology on $\mathbb{R}$. This topology has a number of interesting properties and was investigated by many authors. Among various works devoted to the I-density topology we mention especially book [4] which is a good survey of the subject.

Example 2 Let us consider another particular case of the Wilczynski construction. Namely, let us put $T=T_{d}$ where $T_{d}$ is the density topology on $\mathbb{R}$. Of course, $T$ satisfies conditions (1), (2) and (3). It is easy to check that $\Phi_{d}(X)=\Phi_{T}(X)$ for each subset $X$ of $\mathbb{R}$ having the Baire property with respect to $T$. Thus we see that $T^{*}=T=T_{d}$, i.e. the density topology $T_{d}$ can be considered as a particular case of the Wilczynski topology.

The following question arises in a natural way.

Question 1 Does there exist a topology $T$ on $\mathbb{R}$ such that

(a) T satisfies conditions (1), (2) and (3),

(b) all Lebesgue measurable subsets of $\mathbb{R}$ have the Baire property with respect to $T$,

(c) there is a subset $X$ of $\mathbb{R}$ having the Baire property with respect to $T$ and also possessing exactly one $K(\mathbb{R}, T)$-density point on $\mathbb{R}$ ? 
This question was posed by Wilczynski (oral communication), and the aim of the present paper is to give an affirmative answer to it. We shall show that the solution of the above Question of Wilczynski can be obtained by using a certain invariant extension $\mu$ of Lebesgue measure $l$ and exploiting the von Neumann topology associated with $\mu$. We want to observe here that the required measure $\mu$, besides being invariant with respect to all translations of $\mathbb{R}$, should satisfy some additional properties. Namely, it should be complete and, for any $\mu$-measurable set $Y$ and for any positive integer $n$, the set $n Y$ should also be $\mu$-measurable and the equality $\mu(n Y)=n \mu(Y)$ should hold. Such a situation creates some additional difficulties during the construction of the measure $\mu$. Notice also that the presented construction of $\mu$ differs from the well-known constructions of S. Kakutani and J. Oxtoby (see [6]), of K. Kodaira and S. Kakutani (see [7]), and of others (see, for example, [8]). However, in some details of our construction we essentially use a certain almost invariant partition of $\mathbb{R}$ like the authors mentioned above. Of course, the construction of the measure $\mu$ is mainly made for the purpose of guaranteeing the existence of a set $X$ described in (c).

This paper consists of three parts. In the second part of the paper we construct in detail the required measure $\mu$. In the third part we consider the von Neumann topology $T(\mu)$ associated with $\mu$ and show that it gives us the positive answer to Wilczynski's question.

Throughout the whole paper we use the following notation:

$\omega \quad$ - the first infinite cardinal number,

$\mathfrak{c} \quad$ - the cardinality of the continuum; as a rule, we identify $\mathfrak{c}$ with the first ordinal number having the cardinality of the continuum,

card $(X)$ - the cardinality of a given set $X$,

$\mathbb{N} \quad$ - the set of all natural numbers,

$\mathbb{R} \quad$ - the set of all real numbers,

$l \quad$ - the classical Lebesgue measure on $\mathbb{R}$,

$L \quad$ - the family of all Lebesgue measurable subsets of $\mathbb{R}$,

$\operatorname{dom}(\nu)$ - the domain of a given measure $\nu$; in particular, $\operatorname{dom}(l)=L$,

$\mathbb{R}^{m} \quad-\quad m$-dimensional Euclidean space, where $m \in \mathbb{N}$; hence we have $\mathbb{R}^{1}=\mathbb{R}$,

$\mathbf{D}_{m} \quad$ - the group of all isometric transformations of the space $\mathbb{R}^{m}$,

$l_{m} \quad$ - the classical $m$-dimensional Lebesgue measure on $\mathbb{R}^{m}$,

$L_{m} \quad$ - the family of all Lebesgue measurable subsets of $\mathbb{R}^{m}$, i.e. $L_{m}=$ $\operatorname{dom}\left(l_{m}\right)$. 


\section{Preliminary Lemmas and Remarks}

Let $E$ be an infinite set and let $X$ and $Y$ be two subsets of $E$. We shall say that these subsets are almost identical if

$$
\operatorname{card}(X \triangle Y)<\operatorname{card}(E),
$$

where the symbol $\triangle$ denotes the operation of symmetric difference.

Let $G$ be a group of transformations of the set $E$ (i.e. $G$ is a subgroup of the group of all bijective mappings acting from $E$ onto $E$ ). Let $\left\{E_{j}: j \in J\right\}$ be a partition of $E$. We shall say that this partition is almost invariant with respect to the group $G$ if, for each subset $J_{0}$ of $J$ and for each element $g$ of $G$, the sets $\cup\left\{E_{j}: j \in J_{0}\right\}$ and $g\left(\cup\left\{E_{j}: j \in J_{0}\right\}\right)$ are almost identical.

Now, let $X$ be a subset of $E$. We shall say that this subset is almost invariant with respect to the group $G$ if the partition $\{X, E \backslash X\}$ of $E$ is almost invariant with respect to $G$.

At this point, it is worth mentioning that some almost invariant partitions were used by Kakutani and Oxtoby in their famous paper [6] devoted to the construction of a nonseparable invariant extension of Lebesgue measure. Anyway, much earlier, some almost invariant subsets of the real line $\mathbb{R}$ were considered by Sierpinski in several of his works.

We begin this section with the construction of a certain almost invariant partition of $\mathbb{R}$. Let us take as the basic group $G$ of transformations of $\mathbb{R}$ the group of all those affine transformations $g$ of $\mathbb{R}$ which can be represented in the form $g(x)=q x+r \quad(x \in \mathbb{R})$ where $q$ is an arbitrary nonzero rational number and $r$ is an arbitrary real number.

It is clear that

1. $G$ contains the group $\mathbf{D}_{1}$ of all isometric transformations of $\mathbb{R}$,

2. $G$ contains the group of all homotheties of $\mathbb{R}$ with center 0 and with nonzero rational coefficients.

Our first aim is to construct a partition of $\mathbb{R}$ which is almost invariant with respect to the above-mentioned group $G$ and has some additional properties.

Lemma 1 There exist two families of sets $\left\{F_{j}: j \in J\right\}, \quad\left\{J_{\alpha}: \alpha<\mathfrak{c}\right\}$ such that the following conditions hold:

(1) $\operatorname{card}(J)=\mathfrak{c}$,

(2) $\operatorname{card}\left(J_{\alpha}\right)=\mathfrak{c}$ for each ordinal $\alpha<\mathfrak{c}$,

(3) $\left\{J_{\alpha}: \alpha<\mathfrak{c}\right\}$ is a partition of the set $J$, 
(4) for any index $j \in J$, the set $F_{j}$ is closed in $\mathbb{R}$ and $l\left(F_{j}\right)>0$,

(5) for any ordinal $\alpha<\mathfrak{c}$ and for any closed subset $F$ of $\mathbb{R}$ with $l(F)>0$, we have $\operatorname{card}\left(\left\{j \in J_{\alpha}: F_{j}=F\right\}\right)=\mathfrak{c}$.

This lemma is well-known and its proof is not difficult (see, for example, [8]).

Let $\left\{F_{j}: j \in J\right\}$ be a family of closed subsets of $\mathbb{R}$, as in Lemma 1 . Obviously, we can identify the set $J$ of indices with the cardinality of the continuum. Thus we put $\left\{F_{j}: j \in J\right\}=\left\{F_{\beta}: \beta<\mathfrak{c}\right\}$. Let us denote by the symbol $\Gamma_{0}$ the group of all homotheties of $\mathbb{R}$ with center 0 and with nonzero rational coefficients. Of course, we have card $\left(\Gamma_{0}\right)=\omega$. Now, let $\left\{G_{\beta}: \beta<\mathfrak{c}\right\}$ be a family of subgroups of the group $G$ such that

(a) $G_{0}=\Gamma_{0}$,

(b) this family is increasing with respect to inclusion,

(c) the union of this family is equal to $G$,

(d) for each ordinal $\beta<\mathfrak{c}$, we have $\operatorname{card}\left(G_{\beta}\right) \leq \operatorname{card}(\beta)+\omega$.

By the method of transfinite induction, let us define a family $\left\{Z_{\beta}: \beta<\mathfrak{c}\right\}$ of subsets of $\mathbb{R}$ satisfying the following relations:

(1) for each ordinal $\beta<\mathfrak{c}$, the set $Z_{\beta}$ is a $G_{\beta}$-orbit of a point of $\mathbb{R}$,

(2) for each ordinal $\beta<\mathfrak{c}$, the set $Z_{\beta}$ intersects the set $F_{\beta}$,

(3) $\left\{Z_{\beta}: \beta<\mathfrak{c}\right\}$ is a disjoint family.

Suppose that, for an ordinal $\beta<\mathfrak{c}$, a partial family $\left\{Z_{\theta}: \theta<\beta\right\}$ has already been defined. Let us put $Z(\beta)=\cup\left\{Z_{\theta}: \theta<\beta\right\}$. It is clear that $\operatorname{card}\left(G_{\beta}(Z(\beta))\right) \leq \operatorname{card}(\beta)+\omega$. Since $l\left(F_{\beta}\right)>0$, we have $\operatorname{card}\left(F_{\beta}\right)=\mathfrak{c}$. Thus there exists a point $z \in F_{\beta} \backslash G_{\beta}(Z(\beta))$. Let us put $Z_{\beta}=G_{\beta}(z)$. Then $Z_{\beta} \cap F_{\beta} \neq \emptyset$ and $Z_{\beta} \cap Z(\beta)=\emptyset$. Hence, by transfinite induction, we are able to define the whole family $\left\{Z_{\beta}: \beta<\mathfrak{c}\right\}$. Now, taking into account that the original set $J$ of indices is identified with $\mathfrak{c}$, we can write $\left\{Z_{\beta}: \beta<\mathfrak{c}\right\}=\left\{Z_{j}\right.$ : $j \in J\}$. Let $\left\{J_{\alpha}: \alpha<\mathfrak{c}\right\}$ be a partition of the set $J$, as in Lemma 1. For each nonzero ordinal $\alpha<\mathfrak{c}$, let us put $E_{\alpha}=\cup\left\{Z_{j}: j \in J_{\alpha}\right\}$. Finally, put $E_{0}=\mathbb{R} \backslash \cup\left\{Z_{j}: j \in\left(J \backslash J_{0}\right)\right\}$. It is obvious that $\left\{E_{\alpha}: \alpha<\mathfrak{c}\right\}=\left\{E_{j}: j \in J\right\}$ is a partition of the real line $\mathbb{R}$.

Lemma 2 The above-mentioned partition has the following properties: 
(1) it is almost invariant with respect to the group $G$,

(2) for each index $j \in J$, the set $E_{j}$ is $\Gamma_{0}$-invariant, i.e. $\Gamma_{0}\left(E_{j}\right)=E_{j}$,

(3) for each $j \in J$ and for each closed subset $F$ of $\mathbb{R}$ with $l(F)>0$, the equality card $\left(E_{j} \cap F\right)=\mathfrak{c}$ holds; in particular, the set $E_{j}$ is l-thick in $\mathbb{R}$, i.e. the inner Lebesgue measure of the complement of $E_{j}$ is equal to zero.

Lemma 2 can be checked directly, without any difficulty. The construction of the family $\left\{Z_{\beta}: \beta<\mathfrak{c}\right\}$ guarantees that properties (1), (2), (3) formulated in this lemma are valid.

Remark 1 In fact, the method which we used above to define the almost invariant partition $\left\{E_{j}: j \in J\right\}$ of the real line goes back to the well known paper of Kakutani and Oxtoby [6]. Notice that in [6] this method is applied in the case where the basic set $E$ coincides with the one-dimensional torus equipped with the probability Lebesgue measure $\lambda$ invariant with respect to the group of all rotations of the torus. Applying this method, Kakutani and Oxtoby were able to construct a nonseparable invariant extension of the measure $\lambda$. Much later, their method was generalized to some classes of topological groups (see, for instance, [9]).

Now, we shall begin a construction of an extension $\mu$ of the Lebesgue measure $l$. This extension will be invariant with respect to the group of all isometric transformations of $\mathbb{R}$ and, in addition, it will satisfy $\mu(q Y)=q \mu(Y)$ for any positive rational number $q$ and for any $\mu$-measurable subset $Y$ of $\mathbb{R}$.

We start with the partition $\left\{E_{j}: j \in J\right\}$ of the real line $\mathbb{R}$ defined above. First, let us note that, in further considerations, it is convenient for us to identify the set $J$ with the unit segment $[0,1]$. Thus we put

$$
\left\{E_{j}: j \in J\right\}=\left\{E_{t}: t \in[0,1]\right\} .
$$

Let $\lambda$ be the probability Lebesgue measure on the segment $[0,1]$. Actually, $\lambda$ is the restriction of the measure $l$ to this segment. Let us define a mapping $\phi: \mathbb{R} \rightarrow \mathbb{R} \times[0,1]$ by $\phi(x)=(x, t(x)) \quad(x \in \mathbb{R})$, where $t(x)=t$ is the unique real number from the segment $[0,1]$ for which $x \in E_{t}$.

Lemma 3 The mapping $\phi$ has the following properties:

(1) $\operatorname{pr}_{2}(\phi(q x))=p r_{2}(\phi(x))$ for each point $x \in \mathbb{R}$ and for every nonzero rational number $q$ (where $\mathrm{pr}_{2}$ denotes the projection into the second coordinate), 
(2) the range of $\phi$ is an $(l \times \lambda)$-thick subset of the product measure space $(\mathbb{R}, l) \times([0,1], \lambda)$.

Proof. Relation (1) holds, since, for each $t \in[0,1]$, we have the equality $\Gamma_{0}\left(E_{t}\right)=E_{t}$.

Now, let us consider the subset $\phi(\mathbb{R})$ of the product $\mathbb{R} \times[0,1]$. For any $t \in[0,1]$, the section of $\phi(\mathbb{R})$ corresponding to $t$ is the set $E_{t}$. But $E_{t}$ is an $l$-thick subset of $\mathbb{R}$. Hence, using the Fubini theorem, we see that $\phi(\mathbb{R})$ is an $(l \times \lambda)$-thick subset of $\mathbb{R} \times[0,1]$; so relation (2) holds.

The last relation is fundamental for what follows. Let us introduce the class $S=\left\{\phi^{-1}(Z): Z \in \operatorname{dom}(l \times \lambda)\right\}$ of subsets of $\mathbb{R}$. Obviously, this class is a $\sigma$-algebra of sets. If $X \in S$, then there exists a set $Z \in \operatorname{dom}(l \times \lambda)$ such that $X=\phi^{-1}(Z)$. Put $\mu(X)=(l \times \lambda)(Z) \quad(X \in S)$. The value $\mu(X)$ is well defined since the range of the mapping $\phi$ is an $(l \times \lambda)$-thick set in $\mathbb{R} \times[0,1]$. By a standard argument (see, for example, [8]), it can also be established that the functional $\mu$ is a measure on the $\sigma$-algebra $S$. Moreover, the measure $\mu$ extends Lebesgue measure $l$. Indeed, take any Lebesgue measurable subset $X$ of $\mathbb{R}$. Then it is easy to see that $X=\phi^{-1}(X \times[0,1])$. Since $\lambda$ is a probability measure, we have $\mu(X)=(l \times \lambda)(X \times[0,1])=l(X)$; so we get the required result.

Now, our goal is to show that the measure $\mu$ can be extended to a measure on $\mathbb{R}$ invariant under the group of all isometric transformations of $\mathbb{R}$. For this purpose, we need two auxiliary propositions.

Lemma 4 Let $X$ be a set of the form $X=\phi^{-1}(A \times B)$ where $A$ is a subset of $\mathbb{R}$ and $B$ is a subset of $[0,1]$. Let $g$ be an arbitrary element of the group $G$. Then the sets $g(X)$ and $\phi^{-1}(g(A) \times B)$ are almost identical.

Proof. Indeed, it can be checked directly that $X=A \cap\left(\cup\left\{E_{t}: t \in B\right\}\right)$. Hence we have $g(X)=g(A) \cap g\left(\cup\left\{E_{t}: t \in B\right\}\right)$. But we also have

$$
\phi^{-1}(g(A) \times B)=g(A) \cap\left(\cup\left\{E_{t}: t \in B\right\}\right) .
$$

Now, using the fact that the partition $\left\{E_{t}: t \in[0,1]\right\}$ of the real line is almost invariant with respect to the group $G$, we obtain the required result.

Lemma 5 Let $Z$ be an arbitrary $(l \times \lambda)$-measurable set in $\mathbb{R} \times[0,1]$ with $(l \times \lambda)(Z)>0$. Then $\operatorname{card}\left(\phi^{-1}(Z)\right)=\mathfrak{c}$.

Proof. Indeed, applying the Fubini theorem, we see that there exists a subset $P$ of the segment $[0,1]$, satisfying the following conditions:

(1) $P$ is a $\lambda$-measurable set, 
(2) $\lambda(P)>0$; in particular, card $(P)=\mathfrak{c}$,

(3) for each $t \in P$, we have $Z(t) \in \operatorname{dom}(l), \quad l(Z(t))>0$, where $Z(t)$ denotes the section of the set $Z$, corresponding to the point $t$.

Fix $t \in P$. Since $l(Z(t))>0$ and the set $E_{t}$ is $l$-thick in $\mathbb{R}$, the set $Z(t) \cap E_{t} \neq \emptyset$. Hence there exists an element $x \in Z(t) \cap E_{t}$. But it is clear that $t=t(x)$, $(x, t) \in Z$ and $x \in \phi^{-1}(Z)$. Thus, for each $t \in P$, we have $E_{t} \cap \phi^{-1}(Z) \neq \emptyset$.From this relation we deduce that card $\left(\phi^{-1}(Z)\right)=\mathfrak{c}$, and the lemma is proved.

Let us introduce the class of sets

$$
S^{*}=\{Y \subseteq \mathbb{R}: \text { there exists } X \in S \text { such that } \operatorname{card}(X \triangle Y)<\mathfrak{c}\}
$$

Lemma 6 For the class $S^{*}$, the next two relations hold:

(1) $S^{*}$ is a $\sigma$-algebra of subsets of $\mathbb{R}$ containing the original $\sigma$-algebra $S$,

(2) $S^{*}$ is a $G$-invariant class of sets; i.e., for each set $Y \in S^{*}$ and for each transformation $g \in G$, we have $g(Y) \in S^{*}$.

Proof. The first relation is trivial. The second can be deduced from Lemma 4 by a standard argument.

Lemma 7 The measure $\mu$ can be uniquely extended to a measure defined on the $\sigma$-algebra $S^{*}$ and invariant with respect to the group of all isometric transformations of $\mathbb{R}$.

This lemma is an easy consequence of the preceding lemmas.

We preserve the same symbol $\mu$ for the measure extended in such a way. Moreover, in our further considerations we use the same symbol to denote the usual completion of the extension mentioned above.

Lemma 8 Let $q$ be an arbitrary positive rational number and let $Y$ be an arbitrary $\mu$-measurable subset of $\mathbb{R}$. Then the set $q Y$ is also $\mu$-measurable and we have $\mu(q Y)=q \mu(Y)$.

Proof. Without loss of generality we may assume that $q \neq 0$, and that $Y$ belongs to the $\sigma$-algebra $S$. Thus there exists a set $Z \in \operatorname{dom}(l \times \lambda)$ such that $Y=\phi^{-1}(Z)$. Now, consider the bijective Borel mapping $f: \mathbb{R} \times[0,1] \rightarrow$ $\mathbb{R} \times[0,1]$ defined by $f(x, t)=(q x, t) \quad(x \in \mathbb{R}, \quad t \in[0,1])$. Taking into account the fact that $t(q x)=t(x) \quad(x \in \mathbb{R})$, we can write $q Y=\phi^{-1}(f(Z))$. Clearly, the set $f(Z)$ is $(l \times \lambda)$-measurable. Therefore, applying the Fubini theorem, we conclude that

$$
\mu(q Y)=(l \times \lambda)(f(Z))=q(l \times \lambda)(Z)=q \mu(Y)
$$


and our lemma is proved.

The next remark is not necessary for what follows, but it is of independent interest.

Remark 2 The method used above also makes it possible to construct a nonseparable analogue of the measure $\mu$. Indeed, let us take the Tychonoff cube $[0,1]^{\mathfrak{c}}$ with the topological weight $\mathfrak{c}$. Equip this cube with the probability product measure $\lambda_{\mathfrak{c}}$. It is known that there exists a $\lambda_{\mathfrak{c}}$-thick subset $J$ of this cube with card $(J)=\mathfrak{c}$ (actually, the existence of $J$ follows from the fact that the cube is a separable topological space). Moreover, we can assume that card $(J \cap Z)=\mathfrak{c}$ for each Borel subset $Z$ of $[0,1]^{\mathfrak{c}}$ having a strictly positive $\lambda_{\mathfrak{c}}$-measure. Now, we take the partition $\left\{E_{j}: j \in J\right\}$ of $\mathbb{R}$ considered above and define a mapping $\psi: \mathbb{R} \rightarrow \mathbb{R} \times[0,1]^{\mathfrak{c}}$ by the formula $\psi(x)=(x, j(x)) \quad(x \in \mathbb{R})$. It can easily be shown, by applying the Fubini theorem, that the range of the mapping $\psi$ is an $\left(l \times \lambda_{\mathfrak{c}}\right)$-thick subset of the product space $\mathbb{R} \times[0,1]^{\mathfrak{c}}$. Starting with this mapping, we introduce the class of sets $S=\left\{\psi^{-1}(Z): Z \in \operatorname{dom}\left(l \times \lambda_{\mathfrak{c}}\right)\right\}$. Obviously, this class is a $\sigma$-algebra of subsets of $\mathbb{R}$. If we put $\nu\left(\psi^{-1}(Z)\right)=\left(l \times \lambda_{\mathfrak{c}}\right)(Z)$ for every $\left(l \times \lambda_{\mathfrak{c}}\right)$-measurable set $Z$, then we obtain a measure $\nu$ on $S$ extending the Lebesgue measure $l$. Using an argument similar to the above one, we prove that the measure $\nu$ can be uniquely extended to a measure invariant with respect to the group $\mathbf{D}_{1}$ of all isometric transformations of $\mathbb{R}$. Moreover, we have $\nu(q Y)=q \nu(Y)$ for every positive rational number $q$ and for every $\nu$-measurable set $Y$. Finally, we see that $\nu$ is a nonseparable measure on $\mathbb{R}$. More precisely, the topological weight of the metric space canonically associated with $\nu$ is equal to the cardinality of the continuum.

Remark 3 Let us take $m$-dimensional Euclidean space $\mathbb{R}^{m}$ equipped with the group $\mathbf{D}_{m}$ of all its isometric transformations. Denote by $\Gamma_{0}$ the group of all homotheties of $\mathbb{R}^{m}$ with center 0 and with nonzero rational coefficients. Let $G$ be the group of transformations of $\mathbb{R}^{m}$ generated by the set $\mathbf{D}_{m} \cup \Gamma_{0}$. Then we can construct a partition $\left\{E_{j}: j \in J\right\}$ of the space $\mathbb{R}^{m}$ almost invariant with respect to $G$ and consisting of $l_{m}$-thick subsets of $\mathbb{R}^{m}$. Using this partition, we define a $\sigma$-algebra of subsets of $\mathbb{R}^{m}$ containing the class $L_{m}$ and invariant under the group $G$. On this $\sigma$-algebra we define a measure $\mu_{m}$ which is an $m$ dimensional analogue of the measure $\mu$, i.e. $\mu_{m}$ has the properties analogous to those of $\mu$. Moreover, taking account of Remark 2, we may even assume that $\mu_{m}$ is a nonseparable measure. (More precisely, we may assume that the weight of the metric space associated with $\mu_{m}$ is equal to c.) 


\section{The Main Result}

Let $E$ be a nonempty set, let $S$ be a $\sigma$-algebra of subsets of $E$ and let $\nu$ be a nonzero complete $\sigma$-finite measure defined on $S$. The well-known von Neumann-Maharam theorem states that there exists at least one topology $T(\nu)$ on $E$ satisfying the following relations:

(1) $T(\nu)$ is a Baire space topology with the countable chain condition (ccc), i.e. each disjoint subfamily of $T(\nu)$ is at most countable,

(2) the ideal of all first category sets with respect to $T(\nu)$ coincides with the ideal of all $\nu$-measure zero sets,

(3) the $\sigma$-algebra of all sets having the Baire property with respect to $T(\nu)$ coincides with $S$.

We say that $T(\nu)$ is the von Neumann topology associated with the original measure $\nu$. Notice that, in general, this topology is not unique for the measure $\nu$ (about the von Neumann topology see, for example, [1] and also Chapter 22 of the Handbook of Boolean Algebras, 1989, Ed. J. D. Monk with R. Bonnet).

Suppose also that a group $G$ of transformations of $E$ is given, and that our measure $\nu$ is invariant (or, more generally, quasiinvariant) under $G$. Then it is obvious that $G$ preserves both the ideal of all first category sets with respect to the topology $T(\nu)$ and the class of all sets having the Baire property with respect to the same topology.

Now, let $G$ be again the group of transformations of the real line $\mathbb{R}$ generated by the union $\mathbf{D}_{1} \cup \Gamma_{0}$. Returning to the measure $\mu$ constructed in the preceding section, we can take the von Neumann topology $T(\mu)$ associated with $\mu$. We see that for this topology the following relations are true:

(1) all Lebesgue measurable subsets of $\mathbb{R}$ have the Baire property with respect to $T(\mu)$,

(2) the class $K(\mathbb{R}, T(\mu))$ is invariant with respect to $G$,

(3) the class $B(\mathbb{R}, T(\mu))$ is invariant with respect to $G$.

Let $Y$ be a $\mu$-measurable subset of $\mathbb{R}$ and let $y$ be a point of $\mathbb{R}$. We say that $y$ is a density point of $Y$ with respect to the measure $\mu$ if

$$
\lim _{n \rightarrow \infty} n \mu(Y \cap[y-1 / n, y+1 / n])=2 .
$$

In particular, if the point $y$ coincides with 0 and is a density point of $Y$, then we have $\lim _{n \rightarrow \infty} n \mu(Y \cap[-1 / n, 1 / n])=2$. Since our measure $\mu$ satisfies the 
condition $\mu(q Y)=q \mu(Y)$ for every positive rational number $q$ and for every $\mu$-measurable set $Y$, we see that 0 is a density point of $Y$ with respect to $\mu$ if and only if $\lim _{n \rightarrow \infty} \mu(n Y \cap[-1,1])=2$. Now, applying the Riesz theorem on convergence in measure, we obtain the following result.

Lemma 9 Let $Y$ be an arbitrary $\mu$-measurable subset of $\mathbb{R}$ and let $y \in \mathbb{R}$. Then the following are equivalent:

(1) $y$ is a density point of the set $Y$ with respect to the measure $\mu$,

(2) y is a $K(\mathbb{R}, T(\mu))$-density point of the set $Y$, i.e. $y$ is a density point of $Y$ in the Wilczynski sense with respect to the von Neumann topology $T(\mu)$ associated with $\mu$.

Lemma 10 There exists a $\mu$-measurable subset $X$ of $\mathbb{R}$ such that 0 is the unique density point of $X$ with respect to the measure $\mu$.

Proof. Endow the product space $\mathbb{R} \times[0,1]$ with the product measure $l \times \lambda$. Of course, we can suppose that this space is a subspace of the Euclidean plane $\mathbb{R}^{2}$. Let $Z$ be the convex hull of $\{(1,1 / 2),(-1,1 / 2),(0,0),(0,1)\}$. Obviously, $Z$ is an $(l \times \lambda)$-measurable subset of $\mathbb{R} \times[0,1]$. Put $X=\phi^{-1}(Z)$. We assert that the set $X$ is the required one. Indeed, fix any point $x$ of $\mathbb{R}$. Then it is not difficult to check that $\lim _{n \rightarrow \infty}(n \mu(X \cap[x-1 / n, x+1 / n])) / 2$ exists and is equal to $\lim _{n \rightarrow \infty}\left(n(l \times \lambda)\left(Z \cap Z_{n}\right)\right) / 2$, where $Z_{n}$ denotes the rectangle $[x-1 / n, x+1 / n] \times[0,1]$. But it is clear that the second limit is equal to $\max \{0, \quad 1-|x|\}$. Hence only the point 0 is a density point of the set $X$ with respect to the measure $\mu$.

Remark 4 Let $k \in \mathbb{N}$. By Lemma 10 we deduce that there exists a $\mu$ measurable subset $X_{k}$ of $\mathbb{R}$ having exactly $k$ density points with respect to $\mu$. Also, it is easy to see that there exists a $\mu$-measurable set $X_{\omega}$ such that the cardinality of the set of density points of $X_{\omega}$ with respect to $\mu$ is $\omega$. Finally, there exists a $\mu$-measurable subset $Y$ of $\mathbb{R}$ with $\mu(Y)>0$ such that no point of $\mathbb{R}$ is a density point for $Y$ with respect to $\mu$.

Lemmas 9 and 10 immediately give us the desired result concerning the question posed by Wilczynski. Namely, we have the following theorem.

Theorem 1 There is a topology $T$ on $\mathbb{R}$ satisfying the following conditions:

(1) $T$ is a Baire space topology with ccc, 
(2) all Lebesgue measurable subsets of $\mathbb{R}$ have the Baire property with respect to $T$,

(3) the classes $K(\mathbb{R}, T)$ and $B(\mathbb{R}, T)$ are invariant under the group $G$,

(4) there exists a subset $X$ of $\mathbb{R}$ having the Baire property with respect to $T$ and possessing exactly one density point in the sense of Wilczynski.

Remark 5 Taking Remark 3 of the preceding section into account, we can conclude that the proposition analogous to Theorem 1 is true for $m$-dimensional Euclidean space $\mathbb{R}^{m}$ where $m \geq 2$.

Remark 6 In [10] it is proved that there exists a measure $\nu$ on $\mathbb{R}$ satisfying the following conditions:

(1) $\nu$ extends the Lebesgue measure $l$,

(2) $\nu$ is invariant under the group $\mathbf{D}_{1}$,

(3) $\nu$ has the uniqueness property on its domain, i.e. each $\sigma$-finite measure defined on $\operatorname{dom}(\nu)$ and invariant under all translations of $\mathbb{R}$ is proportional to $\nu$,

(4) there exists a $\nu$-measurable subset of $\mathbb{R}$ having exactly one density point with respect to $\nu$.

\section{References}

[1] J. Oxtoby, Measure and Category, Springer Verlag, New York, 1980, (2nd ed.).

[2] F. Tall, The density topology, Pacific Journal of Mathematics, 62 (1976), $275-284$.

[3] W. Wilczynski, A generalization of density topology, Real Analysis Exchange, 8 (1982-1983), 16-20.

[4] K. Ciesielski, L. Larson and K. Ostaszewski, I-density Continuous Functions, Memoirs of Amer. Math. Soc., 515, 1994.

[5] W. Wilczynski, A category analogue of the density topology, approximate continuity, and the approximate derivative, Real Analysis Exchange, 10 (1984-1985), 241-265. 
[6] S. Kakutani and J. Oxtoby, Construction of a nonseparable invariant extension of the Lebesgue measure space, Ann. Math., 52 (1950), 580590 .

[7] K. Kodaira and S. Kakutani, A nonseparable translation invariant extension of the Lebesgue measure space, Ann. Math., 52 (1950), 574-579.

[8] A. B. Kharazishvili, Invariant Extensions of the Lebesgue measure, Izd. Tbil. Gos. Univ., Tbilisi, (1983) (in Russian).

[9] E. Hewitt and K. Ross, Abstract Harmonic Analysis, vol. 1, SpringerVerlag, Berlin, (1963).

[10] A. B. Kharazishvili, Some remarks on density points and the uniqueness property for invariant extensions of the Lebesgue measure, Acta Universitatis Carolinae, Mathematica et Physica, 35 no. 2 (1994), 33-39. 